\title{
Bioinformatics analyses combined microarray identify the deregulated microRNAs in oral cancer
}

\author{
JING CUI ${ }^{1,2}$, DALU LI $^{3}$, WENMEI ZHANG $^{3}$, LIANG SHEN $^{4}$ and XIN XU ${ }^{2}$ \\ ${ }^{1}$ Department of Oral and Maxillofacial Surgery, Jinan Stomatologic Hospital, Jinan, Shandong 250010; \\ ${ }^{2}$ School of Stomatology, Shandong University; ${ }^{3}$ Department of Oral Surgery, Jinan Stomatological Hospital; \\ ${ }^{4}$ Department of Obstetrics and Gynecology, Shandong Provincial Hospital Affiliated to Shandong University, \\ Jinan, Shandong 250010, P.R. China
}

Received October 19, 2013; Accepted March 20, 2014

DOI: $10.3892 / \mathrm{ol} .2014 .2070$

\begin{abstract}
MicroRNAs (miRNAs) are important in the regulation of cell growth, differentiation, apoptosis and carcinogenesis. The overexpression of oncogenic miRNAs or the underexpression of tumor suppressor miRNAs exhibits a critical function in the tumorigenesis of oral cancer. The aim of the present study was to identify differentially expressed miRNAs (DE-miRNAs), which may differentiate oral cancer from normal tissues, as well as the molecular signatures that differ in tumor histology. The miRNA expression profiles of GSE28100 [the Gene Expression Omnibus (GEO) accession number] were downloaded from the GEO database and an independent sample t-test was used to identify statistical differences between the DE-miRNAs of the oral cancer patients and the healthy control subjects. The target genes of DE-miRNA were retrieved from the miRecords database. Furthermore, a protein-protein interaction network was constructed using the Search Tools for the Retrieval of Interacting Genes database and Cytoscape software. A total of 15 DE-miRNAs were identified and among them, hsa-miR-15a drew specific attention. Gene Ontology analysis revealed that the target genes of fibroblast growth factor (FGF)2 are involved in the progression of oral cancer. Furthermore, functional analysis indicated that the FGF-receptor signaling pathway was significantly upregulated in oral cancer. hsa-miR-15a is important in the regulation of oral cancer and thus, may present a potential biomarker for the prediction of oral cancer progression.
\end{abstract}

\section{Introduction}

Oral cancer is the sixth most common cancer worldwide, with a high prevalence in regions where individuals habitually smoke cigarettes and consume alcohol (1). In addition, the five-year

Correspondence to: $\mathrm{Dr} \mathrm{Xin} \mathrm{Xu}$, School of Stomatology, Shandong University, 44 Wenhuaxilu, Jinan, Shandong 250100, P.R. China

E-mail: xinxu2005@163.com

Key words: microRNA, oral cancer, hsa-miR-15a relative survival rate of distant metastasis for oral cancer is $\sim 30-40 \%$ (2). In the USA, 41,380 individuals are diagnosed with oral cancer annually and 7,890 individuals succumbed to the disease in 2012 (3).

MicroRNAs (miRNAs) are a family of endogenous, non-coding, 22-25 nt RNAs that regulate target mRNA. Accumulating evidence indicates that miRNAs are involved in important biological processes associated with apoptosis, proliferation, differentiation, angiogenesis and metastasis. Therefore, the deregulation of such processes may exhibit an effect on cancer initiation, progression and treatment outcome $(4,5)$.

It is hypothesized that miRNAs may serve as valuable tools in cancer diagnosis. Previous studies using miRNA microarray analysis have identified statistically unique profiles, which may discriminate cancer samples from healthy control samples (6). Siow et al (7) used an miRNA microarray to identify the differentially expressed miRNAs (DE-miRNAs) between oral squamous cell carcinoma and non-cancer cells, and miR-31 and miR-375 were found to significantly correlate with clinicopathological parameters.

The aim of the present study was to identify the miRNAs, which may be important in the progression of oral cancer and to analyze their involvement in this process. An independent sample t-test was used to analyze the raw data in order to obtain credible data of the DE-miRNAs. In addition, an interaction network was constructed using the Search Tools for the Retrieval of Interacting Genes (STRING) database and Cytoscape software. The results of the current study support the hypothesis that miRNA expression is deregulated in oral cancer patients compared with healthy individuals.

\section{Materials and methods}

Microarray analysis. The miRNA expression profile of GSE28100 [the Gene Expression Omnibus (GEO) accession number] was downloaded from the GEO database, which was collected by Jung et al (8). The expression data of miRNAs was obtained using the GEO accession number, GSE28100, with the purpose of identifying aberrantly expressed miRNAs in oral squamous cell carcinomas. The expression profiles of miRNAs in 17 patients with oral cancer and three healthy control subjects were available. 
Identification of DE-miRNAs. The raw data were transformed into identifiable expression data and the missing data was completed. Background corrections and quartile data normalization were performed with the robust multi-array average using the default parameters in the affy package. In addition, the data were analyzed using BRB-ArrayTools version 4.2 (National Cancer Institute; http://linus.nci.nih. gov/BRB-ArrayTools.html).

Predicting the target genes of DE-miRNAs. The miRecords database (http://miRecords.umn.edu/miRecords), which is a resource for animal miRNA-target interactions, was used to analyze the target genes of the DE-miRNAs. miRecords integrates the predicted targets of the following miRNA target prediction tools: DIANA-microT (http://diana.csla- b.ece.ntua. gr/microT), MicroInspector (http://bioinfo.uni-plovdiv. bg/microinspector), miRanda (http://www.microrna. org/microrna/home.do), MirTarget2 (http://mirdb.org/miRDB), miTarget $^{\mathrm{TM}}$ (http://cbit.snu.ac.kr/ miTarget), NBmiRTar (http://wotan.wistar.upenn.edu/NBmiRTar/login.php), PicTar (http://pictar.bio.nyu.edu), PITA (http://genie.weizmann. ac.il/index.html), RNA22 (http:/cbcsrv.watson.ibm.com/rna22. html), RNAhybrid (http://bibiserv.techfak.uni-bielefeld. de/rnahybrid) and TargetScan (http://www.targetscan.org). The genes that were predicted by at least five of the 10 databases were selected as DE-miRNA targets for subsequent analysis to reduce the quantity of false-positive results.

Network analysis and functional annotation. The STRING database includes experimental and predicted interaction information and STRING version 9.1 comprises of $>1,100$ completely sequenced organisms (9). To identify the interactive associations between the target genes and other genes, the target genes of DE-miRNAs were input into STRING. The Cytoscape software was used to visualize these associations and the mined modules.

The Database for Annotation, Visualization and Integrated Discovery (DAVID) includes a broad selection of functional annotation tools for understanding the biological significance of numerous genes. In the current study, DAVID was used to label the function of genes within the modules and the Gene Ontology (GO) terms with an adjusted P-value of $<0.05$ and a count $>2$ were selected.

Statistical analysis. An independent sample t-test was used to identify the DE-miRNAs between the oral cancer patients and healthy control subjects, and $\mathrm{P}<0.001$ was considered to indicate a statistically significant difference.

\section{Results}

Identification of DE-miRNAs. miRNA expression data was obtained using the accession number, GSE28100, and included 17 patients with oral cancers and three healthy control subjects. The miRNA expression data was analyzed by BRB-ArrayTools and 15 miRNAs exhibited significant differential expression $(\mathrm{P}<0.001$; Table I).

Target gene prediction. Since miRNAs regulate the post-transcriptional regression of target genes, the putative target genes
Table I. Differentially expressed miRNAs obtained using Gene Expression Omnibus accession number, GSE28100.

\begin{tabular}{llrc}
\hline $\mathrm{n}$ & \multicolumn{1}{c}{ miRNA } & $\begin{array}{c}\text { Fold } \\
\text { change }\end{array}$ & P-value \\
\hline 1 & hsa-miR-424 & 10.31 & 0.0000650 \\
2 & hsa-miR-21 & 6.34 & 0.0002210 \\
3 & hsa-miR-15b & 7.81 & 0.0006760 \\
4 & hsa-miR-923 & 0.21 & 0.0001064 \\
5 & hsa-miR-146b-5p & 5.54 & 0.0001082 \\
6 & hsa-miR-331-3p & 2.92 & 0.0001400 \\
7 & hsa-miR-15a & 3.58 & 0.0001929 \\
8 & hsa-miR-26b & 5.99 & 0.0003248 \\
9 & hsa-miR-455-3p & 5.18 & 0.0003526 \\
10 & hsa-let-7f & 6.49 & 0.0003598 \\
11 & hsa-miR-27a & 2.83 & 0.0005683 \\
12 & hsa-miR-96 & 5.08 & 0.0006193 \\
13 & hsa-miR-590-5p & 3.00 & 0.0007849 \\
14 & hsa-miR-28-5p & 4.68 & 0.0008894 \\
15 & hsa-let-7a & 4.16 & 0.0009845
\end{tabular}

$\mathrm{P}<0.001$.

of DE-miRNAs were retrieved from miRecords, which selects the target genes that have been retrieved by at least five databases. Furthermore, these target genes were searched for using PubMed and a large list of target genes were confirmed to be associated with oral cancer (Table II). Among these genes, hsa-miR-15a had the greatest number of target genes associated with oral cancer.

Interaction network construction and module analysis. The target genes of 12 DE-miRNAs were input into the STRING database, which identified the significant interactions with a confidence score of $>0.9$. In addition, a protein-protein interaction (PPI) network was constructed using Cytoscape software (Fig. 1).

The PPI network reveals the molecular mechanisms of oral cancer, however, it contains numerous nodes and interactions, which makes it difficult to select the useful information. Therefore, the modules were mined in the PPI network to include insulin-like growth factor (IGF)2-receptor (R), cluster of differentiation 44, IGF2, IGF1-R and fibroblast growth factor (FGF)2. Functional analysis demonstrated that the genes in this module could be divided into 21 functional GO terms (the 10 most significant terms are shown in Table III). Among these functional nodes, the most significant GO category was identified to be the FGF-R signaling pathway.

The target genes of FGF2 were identified to be associated with the GO categories of apoptosis, programmed cell death, cell migration, cell death and cell motility.

\section{Discussion}

In the present study, 15 DE-miRNAs were identified to exhibit a regulatory function in the progression of oral cancer. As a 


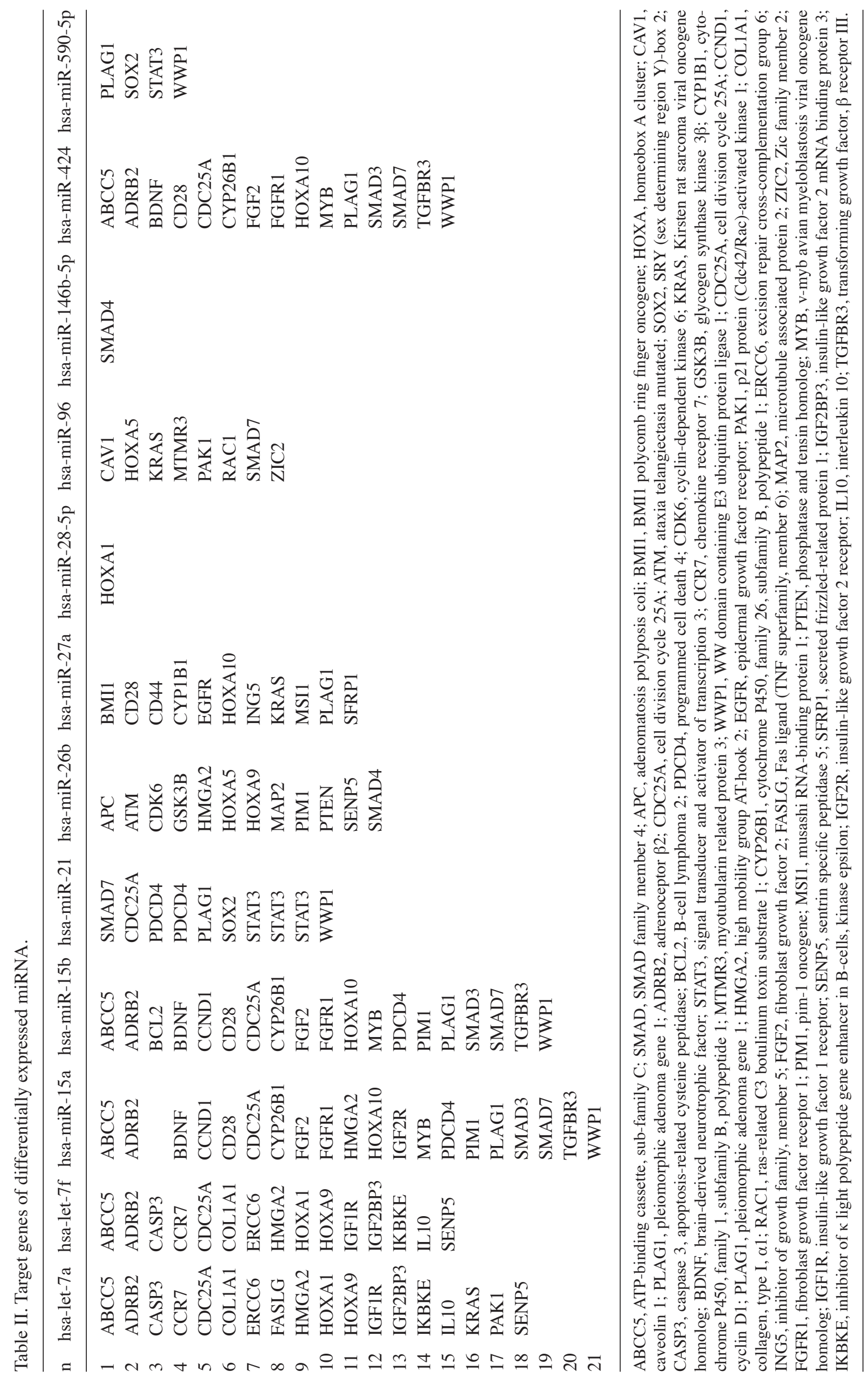


Table III. Gene Ontology analysis of the 10 most significant target genes (False discovery rate, $\mathrm{P}<0.05$ ).

\begin{tabular}{|c|c|c|c|}
\hline $\mathrm{n}$ & Description & P-value & Genes in test set \\
\hline 1 & $\begin{array}{l}\text { Fibroblast growth factor } \\
\text { receptor signaling pathway }\end{array}$ & 0.000004 & CEP5, FGF2, FGFR3 and FGFR4 \\
\hline 2 & $\begin{array}{l}\text { Transmembrane receptor protein } \\
\text { tyrosine kinase signaling pathway }\end{array}$ & 0.000087 & CEP57, FGF2, FGFR3, FGFR4 and IGF1R \\
\hline 3 & $\begin{array}{l}\text { Enzyme-linked receptor } \\
\text { protein signaling pathway }\end{array}$ & 0.000440 & CEP57, FGF2, FGFR3, FGFR4 and IGF1R \\
\hline 4 & Phosphate metabolic process & 0.003100 & FGFR2, FGF2, FGFR2, FGF4, IGF1R, SDC3 \\
\hline 5 & Phosphorus metabolic process & 0.003100 & FGFR2, FGF2, FGFR2, FGF4, IGF1R, SDC3 \\
\hline 6 & Phosphorylation & 0.009800 & FGF2, FGFR3, FGFR4, IGF1R \\
\hline 7 & Positive regulation of cell proliferation & 0.009800 & FGF2, FGFR3, FGFR4, IGF1R \\
\hline 8 & $\begin{array}{l}\text { Cell surface receptor-linked } \\
\text { signal transduction }\end{array}$ & 0.011000 & CEP57, IGF2BP3, FGF2, FGFR3, FGFR4, IGF1R and PLAUR \\
\hline 9 & Wound healing & 0.018000 & CD44, FGF2 and FN1 \\
\hline 10 & Response to wounding & 0.019000 & CD44, IGF2BP3, FGF2 and FN1 \\
\hline
\end{tabular}

CEP, caenorhabditis elegans; FGF, fibroblast growth factor; FGFR, fibroblast growth factor receptor; IGF1R, insulin-like growth factor 1 receptor; SDC3, syndecan-3; IGF2BP3, insulin-like growth factor 2 mRNA binding protein 3; PLAUR, plasminogen activator urokinase receptor; FN1, fibronectin 1 .

A

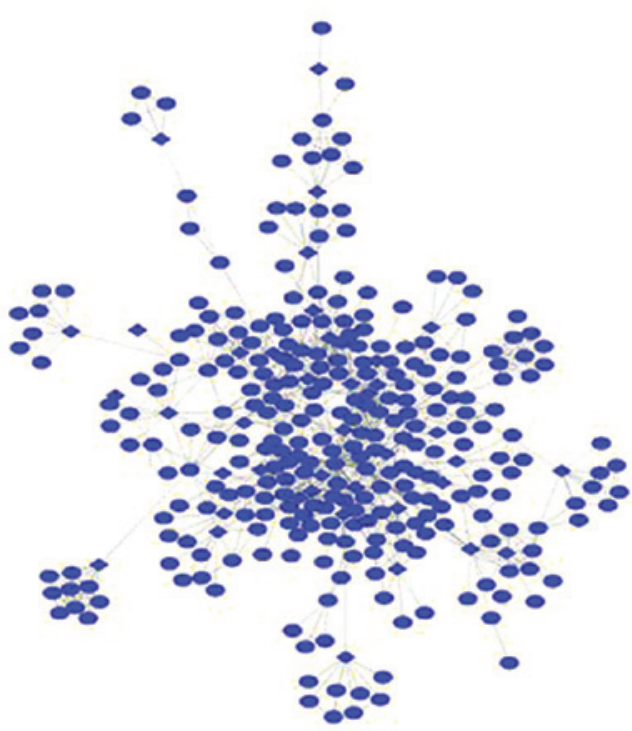

B

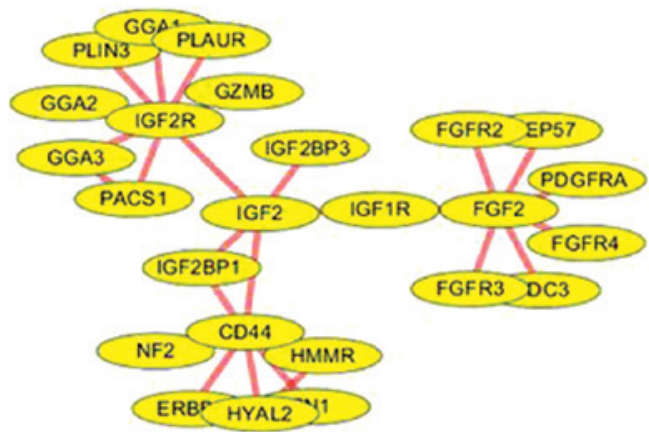

Figure 1. (A) PPI network construction. (B) The module identified from the PPI network. PPI, protein-protein interaction; PLAUR, plasminogen activator urokinase receptor; GGA, golgi-associated, $\gamma$ adaptin ear containing, ARF binding protein; PLIN3, perilipin 3; IGF2R, insulin-like growth factor 2 receptor; GZMB, granzyme B; PACS1, phosphofurin acidic cluster sorting protein 1; IGF2BP, insulin-like growth factor 2 mRNA binding protein; IGF2, insulin-like growth factor 2; NF2, neurofibromin 2; HYAL2, hyaluronoglucosaminidase 2; HMMR, hyaluronan-mediated motility receptor; IGF1R, insulin-like growth factor 1 receptor; FGF2, fibroblast growth factor 2; FGFR, fibroblast growth factor receptor; PDGFRA, platelet-derived growth factor receptor, $\alpha$ polypeptide.

result of retrieving the target genes of the DE-miRNAs from miRecords, the target genes of 12 DE-miRNAs were found to be associated with oral cancer. Through PPI network construction and module analysis, an FGF2 module was formed and was identified to be significant in the progression of oral cancer. Furthermore, functional analysis showed that the module was significantly associated with the FGF-R signaling pathway.

The expression of 12 DE-miRNAs, including hsa-miR-15a, was identified as a possible biomarker to monitor oral cancer progression and early diagnosis. In addition, hsa-miR-15a was found to be downregulated in certain hematological tumors and is considered to regulate cancer-associated genes that influence apoptosis, the cell cycle, proliferation and survival $(10,11)$. In addition, hsa-miR-15a is frequently downregulated in chronic lymphocytic leukemia, prostate cancer and non-small cell lung cancer (12-14), and the inhibition of hsa-miR-15a significantly increases the secreted matrix metalloproteinase- 9 expression in neuroblastoma (15). However, Ricieri et al (16) identified that hsa-miR-15a expression levels were upregulated in the 
majority of oral cancers samples. The results of the current study using the accession number, GSE28100 revealed a 3.58-fold change in hsa-miR-15a expression, compared with that of the healthy control subjects, which indicated that hsa-miR-15a is involved in the progression of oral cancer.

In addition, FGF2 formed a module in the PPI network that was constructed based on oral cancer samples, which indicated that FGF2 has an important function in the progression of oral cancer. FGF2 is an $18-\mathrm{kDa}$ non-glycosylated polypeptide consisting of 146 amino acids (17), which mediates various cellular events, including migration, angiogenesis, motility, proliferation and differentiation $(18,19)$.

In addition, FGF 2 promotes tumor progression and previous studies indicate that the upregulation of FGF2 is important in prostate carcinogenesis and malignant progression (20). FGF2 is one of the most well-studied factors involved in angiogenesis (21). Lau et al (22) identified that the expression of FGF2 decreases E-cadherin levels by upregulating its transcriptional repressors, Slug and ZEB1, in human ovarian cancer cells.

The FGF-R, a sub-family of the superfamily of receptor tyrosine kinases, may regulate human development and metabolism. Previous studies have shown that FGF-R may be important in carcinogenesis $(23,24)$. Furthermore, studies have indicated that FGF-R1 is amplified in $20 \%$ of squamous non-small cell lung cancers (25) and mutations of FGF-R2 have been described in $12 \%$ of endometrial carcinomas (26). Furthermore, $\sim 10 \%$ of gastric cancer cases exhibit FGF-R2 amplification and mutations (27).

In conclusion, the current study identified 15 DE-miRNAs, which may be important in the progression of oral cancer and hsa-miR-15a demonstrated the greatest quantity of target genes. In addition, FGF2 expression was identified to be significantly associated with the presentation of oral cancer. However, further investigation regarding the function of FGF2 is required.

\section{References}

1. Zygogianni AG, Kyrgias G, Karakitsos P, et al: Oral squamous cell cancer: early detection and the role of alcohol and smoking. Head Neck Oncol 3: 2, 2011.

2. Zini A, Czerninski R and Sgan-Cohen HD: Oral cancer over four decades: epidemiology, trends, histology, and survival by anatomical sites. J Oral Pathol Med 39: 299-305, 2010.

3. Siegel R, Naishadham D and Jemal A: Cancer statistics, 2013. CA Cancer J Clin 63: 11-30, 2013.

4. Mirnezami AH, Pickard K, Zhang L, Primrose JN and Packham G: MicroRNAs: key players in carcinogenesis and novel therapeutic targets. Eur J Surg Oncol 35: 339-347, 2009.

5. Ruan K, Fang X and Ouyang G: MicroRNAs: novel regulators in the hallmarks of human cancer. Cancer Lett 285: 116-126, 2009.

6. Cicatiello L, Mutarelli M, Grober OM, et al: Estrogen receptor alpha controls a gene network in luminal-like breast cancer cells comprising multiple transcription factors and microRNAs. Am J Pathol 176: 2113-2130, 2010.

7. Siow M, Karen Ng L, Vincent Chong V, et al: Dysregulation of miR-31 and miR-375 expression is associated with clinical outcomes in oral carcinoma. Oral Dis: Apr 17, 2013 (Epub ahead of print) doi: 10.1111/odi.12118.
8. Jung HM, Phillips BL, Patel RS, et al: Keratinization-associated miR-7 and miR-21 regulate tumor suppressor reversion-inducing cysteine-rich protein with kazal motifs (RECK) in oral cancer. J Biol Chem 287: 29261-29272, 2012.

9. Franceschini A, Szklarczyk D, Frankild S, et al: STRING v9.1: protein-protein interaction networks, with increased coverage and integration. Nucleic Acids Res 41: D808-D815, 2013.

10. Cimmino A, Calin GA, Fabbri M, et al: miR-15 and miR-16 induce apoptosis by targeting BCL2. Proc Natl Acad Sci USA 102: 13944-13949, 2005.

11. Calin GA, Cimmino A, Fabbri M, et al: MiR-15a and miR-16-1 cluster functions in human leukemia. Proc Natl Acad Sci USA 105: 5166-5171, 2008.

12. Urbich C, Kuehbacher A and Dimmeler S: Role of microRNAs in vascular diseases, inflammation, and angiogenesis. Cardiovasc Res 79: 581-588, 2008.

13. Bonci D, Coppola V, Musumeci M, et al: The miR-15a-miR-16-1 cluster controls prostate cancer by targeting multiple oncogenic activities. Nat Med 14: 1271-1277, 2008.

14. Bandi N, Zbinden S, Gugger M, et al: miR-15a and miR-16 are implicated in cell cycle regulation in a $\mathrm{Rb}$-dependent manner and are frequently deleted or down-regulated in non-small cell lung cancer. Cancer Res 69: 5553-5559, 2009.

15. Xin C, Buhe B, Hongting L, et al: MicroRNA-15a promotes neuroblastoma migration by targeting reversion-inducing cysteine-rich protein with Kazal motifs (RECK) and regulating matrix metalloproteinase-9 expression. FEBS J 280: 855-866, 2013.

16. Ricieri Brito JA, Gomes CC, Santos Pimenta FJ, et al: Reduced expression of mir15a in the blood of patients with oral squamous cell carcinoma is associated with tumor staging. Exp Ther Med 1: 217-221, 2010

17. Okada-Ban M, Thiery JP and Jouanneau J: Fibroblast growth factor-2. Int J Biochem Cell Biol 32: 263-267, 2000.

18. Dow JK and deVere White RW: Fibroblast growth factor 2: its structure and property, paracrine function, tumor angiogenesis, and prostate-related mitogenic and oncogenic functions. Urology 55: 800-806, 2000

19. Chalkiadaki G, Nikitovic D, Berdiaki A, et al: Fibroblast growth factor- 2 modulates melanoma adhesion and migration through a syndecan-4-dependent mechanism. Int $\mathrm{J}$ Biochem Cell Biol 41: 1323-1331, 2009.

20. Giri D, Ropiquet F and Ittmann M: Alterations in expression of basic fibroblast growth factor (FGF) 2 and its receptor FGFR-1 in human prostate cancer. Clin Cancer Res 5: 1063-1071, 1999.

21. Cross MJ and Claesson-Welsh L: FGF and VEGF function in angiogenesis: signalling pathways, biological responses and therapeutic inhibition. Trends Pharmacol Sci 22: 201-207, 2001.

22. Lau MT, So WK and Leung PC: Fibroblast growth factor 2 induces E-cadherin down-regulation via PI3K/Akt/mTOR and MAPK/ERK signaling in ovarian cancer cells. PLoS One 8: e59083, 2013.

23. Kelleher FC, O'Sullivan H, Smyth E, McDermott R and Viterbo A: Fibroblast growth factor receptors, developmental corruption and malignant disease. Carcinogenesis 34: 2198-2205, 2013.

24. Liang G, Chen G, Wei X, Zhao Y and Li X: Small molecule inhibition of fibroblast growth factor receptors in cancer. Cytokine Growth Factor Rev 24: 467-475, 2013.

25. Weiss J, Sos ML, Seidel D, et al: Frequent and focal FGFR1 amplification associates with therapeutically tractable FGFR1 dependency in squamous cell lung cancer. Sci Transl Med 2: 62ra93, 2010.

26. Dutt A, Salvesen HB, Chen TH, et al: Drug-sensitive FGFR2 mutations in endometrial carcinoma. Proc Natl Acad Sci USA 105: 8713-8717, 2008.

27. Kunii K, Davis L, Gorenstein J, et al: FGFR2-amplified gastric cancer cell lines require FGFR2 and Erbb3 signaling for growth and survival. Cancer Res 68: 2340-2348, 2008. 